EPJ Web of Conferences 66, 02097 (2014)

DOI: $10.1051 /$ epjconf/ 20146602097

(C) Owned by the authors, published by EDP Sciences, 2014

\title{
Study of the level structure of ${ }^{108} \mathrm{Ag}$
}

J. Sethi ${ }^{1}$,a , R. Palit ${ }^{1, b}$, S. Saha ${ }^{1}$, T. Trivedi ${ }^{1}$, G. H. Bhat ${ }^{2}$, J. A. Sheikh ${ }^{2}$, P. Datta ${ }^{3}$, J. J. Carroll ${ }^{4}$, S. Chattopadhyay $^{5}$, R. Donthi ${ }^{1}$, U. Garg ${ }^{6}$, S. Jadhav ${ }^{1}$, H. C. Jain ${ }^{1}$, S. Karamian ${ }^{7}$, S. Kumar ${ }^{8}$, M. S. Litz $^{4}$, D. Mehta ${ }^{9}$, B. S. Naidu ${ }^{1}$, Z. Naik ${ }^{10}$, S. Sihotra ${ }^{9}$, and P. M. Walker ${ }^{11}$

${ }^{1}$ Tata Institute of Fundamental Research, Mumbai, 400 005, India

${ }^{2}$ Department of Physics, University of Kashmir, Srinagar, 190 006, India

${ }^{3}$ Ananda Mohan College, Kolkata 700 009, India

${ }^{4}$ U S Army Research Laboratory, Adelphi, MD 20783, USA

${ }^{5}$ Saha Institute of Nuclear Physics, Kolkata 700 064, India

${ }^{6}$ University of Notre Dame, Notre Dame, IN 46556, USA

${ }^{7}$ Joint Institute of Nuclear Research, Dubna 141980, Russia

${ }^{8}$ University of Delhi, Delhi 110 007, India

${ }^{9}$ Panjab University, Chandigarh 160 014, India

${ }^{10}$ Sambalpur University, Sambalpur 143 005, India

${ }^{11}$ Department of Physics, University of Surrey, Guildford, Surrey GU2 7XH, UK

\begin{abstract}
The high spin structure of ${ }^{108} \mathrm{Ag}$ nucleus has been studied using the reaction ${ }^{11} \mathrm{~B}+{ }^{100} \mathrm{Mo}$ at $39 \mathrm{MeV}$ with Indian National Gamma Array (INGA) at TIFR-BARC accelerator facility. From the two- and higher-fold coincidence analysis of the emitted $\gamma$-rays, the level structure of the nucleus is built, with addition of around $\sim 60$ new transitions. A new positive parity dipole band has been observed and significant additions have been made in the low spin region. A pair of nearly degenerate, negative parity, dipole bands is established, which is studied using the triaxial projected shell model (TPSM).
\end{abstract}

\section{Introduction}

Investigation of the excited states of odd-odd nuclei near A 110 region continues to provide new insight on novel excitation modes of nuclei, like magnetic rotation, antimagnetic rotation and chiral rotation. In a Relativistic Mean Field (RMF) calculation by Meng et. al, some of the odd-odd isotopes of $\mathrm{Rh}, \mathrm{Ag}$ and In have been predicted to possess multiple chiral bands because of their triaxial shapes [1]. These transitional nuclei, have a character which is in between spherical and axially symmetric deformed nuclei, and can be described using the triaxial deformed mean field [2]. In addition, these nuclei have neutron particles and proton holes in the high $j$-orbitals. The low-lying two-quasiparticle states involving high- $j$ orbitals can lead to low energy isomers [3]. In search of these various interesting phenomena, a detailed spectroscopic study of ${ }^{108} \mathrm{Ag}$ was carried out. ${ }^{108} \mathrm{Ag}$ will be a good laboratory to test the RMF prediction [1]. This nucleus, ${ }^{108} \mathrm{Ag}$, has a very long lived isomer with a half life $T_{1 / 2}=438$ years in the low-spin region. Isomers across the nuclear chart have always been

\footnotetext{
ae-mail: jasmine@tifr.res.in

be-mail: palit@tifr.res.in
}

This is an Open Access article distributed under the terms of the Creative Commons Attribution License 2.0, which permits unrestricted use, distribution, and reproduction in any medium, provided the original work is properly cited. 
a topic of immense interest and such long lived isomers are of particular relevance in exploiting their applications in the field of high energy density storage devices [4]. A spectroscopic measurement of states around the isomer is of vital importance due to its crucial role in estimating the depletion pathway of the isomer and the concerned rate.

\section{Experimental Details}

The high spin states of ${ }^{108} \mathrm{Ag}$ were populated in a heavy ion fusion evaporation reaction ${ }^{100} \mathrm{Mo}\left({ }^{11} \mathrm{~B}, 3 \mathrm{n}\right){ }^{108} \mathrm{Ag}$ at $39 \mathrm{MeV}$ beam energy. The ${ }^{11} \mathrm{~B}$ beam was provided by the Pelletron-LINAC facility at Mumbai. The ${ }^{100} \mathrm{Mo}$ target used was $10 \mathrm{mg} / \mathrm{cm}^{2}$ thick. The $\gamma$-rays from de-excitation of the nuclei populated in the experiment, were detected using the Indian National Gamma Array (INGA) consisting of 18 Compton suppressed clover detectors. Two- and higher-fold clover coincidence events were recorded in a fast digital data acquisition system based on Pixie-16 modules of XIA LLC [5]. The data sorting routine "Multi pARameter time-stamped based COincidence Search program (MARCOS)" developed at TIFR, sorts the time stamped data to generate $E_{\gamma}-E_{\gamma}$ matrices and $E_{\gamma}-E_{\gamma}-E_{\gamma}$ cubes compatible with Radware format [6]. The three-fold coincident data were used to develop the level scheme. The spins and parities of the levels were assigned by using a Directional Correlation of Oriented nuclei (DCO) ratio analysis followed by linear polarization measurements of various transitions.

The multipolarities of $\gamma$-rays were deduced from the angular correlation analysis [7] using the DCO ratio method for two coincident $\gamma$-rays $\gamma_{1}$ and $\gamma_{2}$, given by:

$$
R_{D C O}=\frac{I\left(\gamma_{1}\right) \text { observed at } 157^{\circ} \text { gated on } \gamma_{2} \text { at } 90^{\circ}}{I\left(\gamma_{1}\right) \text { observed at } 90^{\circ} \text { gated on } \gamma_{2} \text { at } 157^{\circ}}
$$

Here, $I\left(\gamma_{1}\right)$ is the intensity of $\gamma_{1}$ measured in coincidence with $\gamma_{2}$. In the present geometry of detectors, the DCO ratios obtained with a stretched quadrupole(dipole) gate are 0.5(1.0) and 1.0(2.0) for pure dipole and quadrupole transitions, respectively. The extracted DCO values were obtained with gates on strong stretched $\Delta I=1$ transitions.

The clover geometry of the detectors present at $90^{\circ}$ was used as a Compton-polarimeter to assign the electric or magnetic nature of $\gamma$-rays $[8,9]$. For a Compton-polarimeter, the polarization asymmetry $\Delta$ of the transition is defined as [8]

$$
\Delta=\frac{a\left(E_{\gamma}\right) N_{\perp}-N_{\|}}{a\left(E_{\gamma}\right) N_{\perp}+N_{\|}}
$$

where, $N_{\perp}\left(N_{\|}\right)$is the number of counts of $\gamma$ transitions scattered perpendicular (parallel) to the reaction plane. A positive value of polarization asymmetry indicates an electric transition while negative value indicates a magnetic transition. The correction factor $a\left(E_{\gamma}\right)$ is a measure of the parallel to perpendicular scattering asymmetry within the crystals of the clover detector.

The level scheme of ${ }^{108} \mathrm{Ag}$ was extended considerably. 64 new $\gamma$-rays were placed in the previously known level scheme [11]. The two-fold coincident, full projection spectrum is shown in Fig. 1. A total of $3.7 \times 10^{8}$ triple coincident events were obtained. A few changes in the placement of energy levels were made, which were confirmed by the newly placed crossover transitions and the connecting transitions from the $E_{\gamma}-E_{\gamma}-E_{\gamma}$ analysis. In the low spin region, substantial changes have been made along with the addition of new $\gamma$ rays near the isomer. The branching ratios of a few low lying transitions in the depleting path were obtained.

Another interesting finding in this nucleus is a pair of negative parity nearly degenerate dipole bands. The yrast band has been established up to spin $19^{-}$along with a partner band up to spin $18^{-}$. 


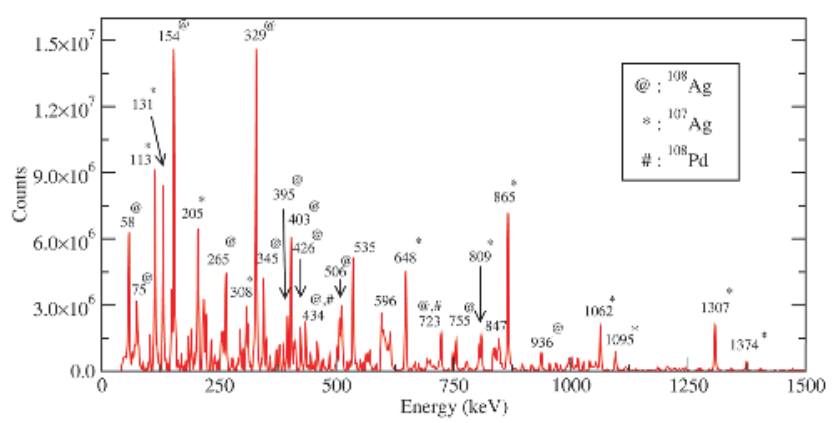

Figure 1. The total projection spectrum, showing the $\gamma$-rays belonging to various nuclei populated in the reaction. The $\gamma$-rays marked with @, * and \# belong to ${ }^{108} \mathrm{Ag},{ }^{107} \mathrm{Ag}$ and ${ }^{108} \mathrm{Pd}$, respectively.

The linking transitions from partner band to yrast band have also been identified. The twin bands have been studied using the Triaxial Projected Shell Model which is discussed in Sec.3 [10].

Two positive parity dipole bands have also been established up to spin $19 \hbar$. One of the bands is a completely new structure based on the presented analysis. The spectrum in Fig.2, generated with sum of two double gates on 265/1118 and 265/1138 kev transitions shows the $\gamma$-rays belonging to the newly observed positive parity band. The second positive parity band already known from the work by Espinoza et. al. [11], has been extended to higher spins. A similar positive parity structure has been observed in ${ }^{112}$ In and reported to be magnetic rotation band [12].

The spins and parities have been assigned to most of the transitions in the level scheme except for the weak ones. The typical DCO ratios and polarization asymmetry values of some of the transitions belonging to ${ }^{108} \mathrm{Ag}$ have been shown in Fig 3 .

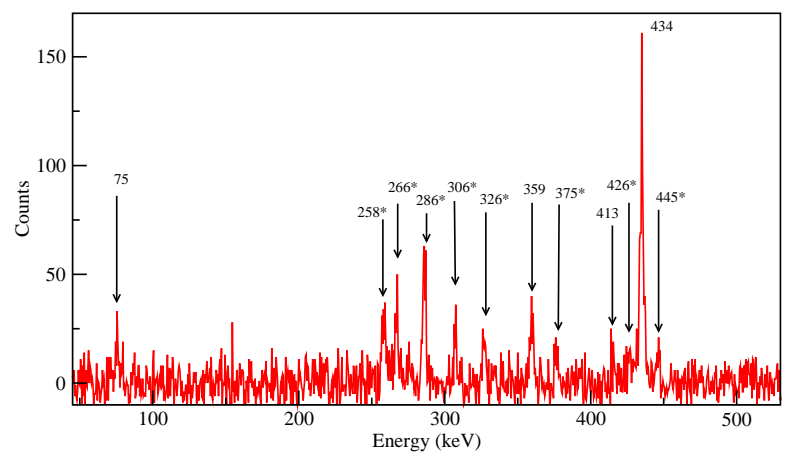

Figure 2. Spectrum with sum of double gates on $265 / 1118$ and $265 / 1138 \mathrm{keV}$ transitions showing the $\gamma$-rays belonging to the newly established positive parity band (which are marked with an $*$ sign).

\section{Triaxial Projected Shell Model}

The newly developed microscopic Triaxial Projected Shell Model (TPSM) approach was used to understand the nature of the observed twin bands [13]. In the TPSM, first a triaxial Nilsson Hamiltonian along with monopole and quadrupole pairing terms is solved in the BCS approach to generate the 


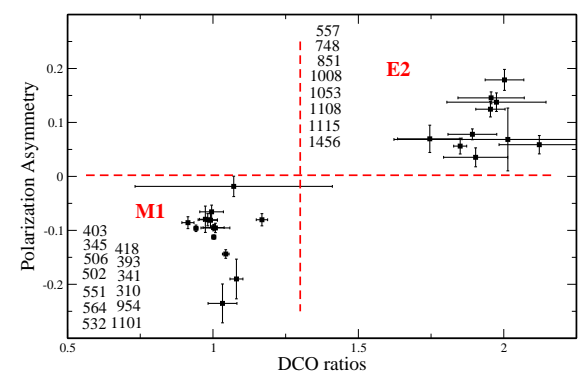

Figure 3. Polarization asymmetry values and DCO ratios for the transitions of the negative parity degenerate bands.

quasi-particle states. Then the three dimensional angular momentum projection operator is used to project out the angular momentum basis states from the intrinsic Nilsson states. In the final step, these angular momentum projected basis states are used to diagonalize the shell model Hamiltonian consisting of the pairing and the quadrupole-quadrupole interactions.

The deformation parameters $\epsilon=0.265$ and $\epsilon^{\prime}=0.09$ were chosen for the present data set, which correspond to a triaxial deformation of $\gamma \sim 19^{\circ}$. The experimentally obtained energies of the yrast and partner bands compared with the theoretically obtained level energies are found to be in good agreement.

\section{Conclusion}

The level scheme of ${ }^{108} \mathrm{Ag}$ has been substantially extended with the addition of around $\sim 60$ new transitions. The spin and parity of different states have been established through angular correlation and polarization measurements. Four bands have been established, two positive parity bands and a pair of nearly degenerate negative parity bands. The negative parity bands are studied using the Triaxial Projected Shell Model and are found to be based on different intrinsic quasi-particle structures. The low spin isomer part has also been modified significantly with addition of a number of new transitions.

\section{References}

[1] J. Meng, J. Peng, S. Q. Zhang, and S. G. Zhou, Phys. Rev. C 73, 037303 (2006).

[2] Peter Möller et. al, Phys. Rev. Lett. 97, 162502 (2006).

[3] H.L. Liu, F.R. Xu, S.W. Xu, R. Wyss and P.M. Walker, Phys. Rev. C 76, 034313 (2007).

[4] P. M. Walker and J. J. Carroll, Phys. Today 58(6), 39 (2005).

[5] R. Palit, et al., Nucl. Instrum. Methods A 680, 90 (2012).

[6] D. C. Radford, Nucl. Instrum. Methods A 361, 297 (1995).

[7] A. Krämer-Flecken et al., Nucl. Instrum. and Methods A 275, 333 (1989).

[8] K. Starosta et al., Nucl. Instrum. Methods A 423, 16 (1999).

[9] R. Palit et al., Pramana 54, 347 (2000).

[10] J. Sethi, et al., Phys. Lett. B 725, 85 (2013).

[11] F. R. Espinoza-Quiñones et. al., Phys. Rev. C 52, 104 (1995).

[12] T. Trivedi, et al., Phys. Rev. C 85, 014327 (2012).

[13] G. H. Bhat, J. A. Sheikh, R. Palit, Phys. Lett. B 707, 250 (2012). 